\title{
BMJ Quality Improving Interprofessional and Coproductive Outcomes of Care for Patients with Chronic Obstructive Pulmonary Disease
}

\author{
Kathleen Moreo, Laurence Greene, Tamar Sapir
}

To cite: Moreo K, Greene L, Sapir T. Improving Interprofessional and Coproductive Outcomes of Care for Patients with Chronic Obstructive Pulmonary Disease. BMJ Quality Improvement Reports 2016;5:u210329.w4679 doi:10.1136/bmjquality. u210329.w4679

Received 8 March 2016 Revised 1 April 2016

PRIME Education, Inc. (PRIME)

Correspondence to Laurence Greene I.greene@primeinc.org

\section{ABSTRACT}

In the U.S., suboptimal care quality for patients with chronic obstructive pulmonary disease (COPD) is reflected by high rates of emergency department visits and hospital readmissions, as well as excessive costs. Moreover, a substantial proportion of COPD patients do not receive guideline-directed therapies. In quality improvement (QI) programs, these types of health care problems are commonly addressed through interventions that primarily or exclusively support physicians in aligning their practices with guidelines and clinical quality measures. However, the root causes of many deficits in health care quality are not necessarily "physician centric." Instead, they often involve suboptimal collaboration among members of interprofessional health care teams and gaps in coproductive relationships among patients and providers.

We conducted a QI project to identify interprofessional and coproductive correlates of COPD care quality in the context of a continuing education program designed to advance knowledge and skill among patients, providers, and the interprofessional COPD team regarding coproductive COPD care. Participants in the program included providers in 30 primary care practices across the U.S. who, along with their own COPD patients and a separate cohort of patients from COPD advocacy groups, completed a patient-provider survey study designed to identify alignments and mismatches in coproductive perceptions and behaviors, a private survey feedback session for each practice's team, and online/mobile educational activities on COPD. In addition, more than 1,000 additional providers and 200 patients participated in just the online/mobile education.

From the patient perspective, baseline measures indicated a high rate of dissatisfaction with COPD treatment plans and suboptimal coproductive interaction with members of the interprofessional health care team. Across providers, there were gaps and variation in provision of patient education, attitudes and practices regarding shared decisionmaking, and care coordination with pulmonary specialists. In addition, relatively low proportions of providers reported high levels of skill in various coproductive processes.
The project outcomes indicated mismatches between COPD patients and providers in perceived ability to recognize COPD exacerbations, shared treatment goals, barriers to medication adherence, perceived impact of COPD on quality of life, and other aspects of COPD care. Providers demonstrated improvements in knowledge and attitudes regarding coproductive and coordinated COPD care.

\section{PROBLEM}

In the U.S., chronic obstructive pulmonary disease (COPD) affects an estimated 24 million adults and accounts for more than 1.5 million emergency department visits and 725,000 hospitalizations per year. The 30-day readmission rate for people hospitalized with acute COPD exacerbations is nearly 25\%.1 2 The total annual cost estimates for COPD exceed $\$ 35$ billion. $^{3}$ Up to $40 \%$ of patients with COPD exacerbations do not receive guideline-directed therapies, and up to $50 \%$ receive at least one medication that is deemed potentially harmful. ${ }^{4} 5$ In quality improvement (QI) programs, these types of health care problems are commonly addressed through interventions, including continuing education, that primarily or exclusively support physicians in aligning their practices with evidence-based guidelines and clinical quality measures. However, the root causes of many deficits in health care quality are not necessarily "physician centric." Instead, they often involve suboptimal collaboration among members of interprofessional healthcare teams and gaps in coproduction, which is defined by the extent to which patients and providers form partnerships in services such as shared treatment goal-setting and decision-making, and assessment of care quality and outcomes. ${ }^{6} 7$

This article reports a phase 1 project designed to identify interprofessional and 
coproductive correlates of COPD care quality in the context of a continuing education program. Conducted by PRIME Education, Inc. (PRIME@), a multi-accredited provider of interprofessional continuing education (IPCE) for health care teams, the project was motivated partly by new models of value-based health care that emphasize aligning practice with patient-centered priorities of the National Quality Strategy (NQS). These priorities include engaging patients and caregivers as partners, promoting effective communication between patients and providers, and enhancing care coordination among members of interprofessional health care teams.

The project involved a patient-provider survey study and educational activities for primary care cliniciansincluding physicians, nurse practitioners, and physician assistants-as well as patients with COPD, and other members of the COPD care team. Our QI team comprised PRIME's trained QI education staff, including specialists in designing patient-provider surveys and analyzing the results and in developing, implementing, and evaluating measurable outcomes of educational interventions that target gaps in care quality. Here we describe how the methods and outcomes of this project have informed the development of an ongoing comprehensive QI education program on COPD.

\section{BACKGROUND}

In addition to high rates of emergency department visits and hospital readmissions, many patients with COPD have long delays in diagnosis and treatment initiation after initial symptoms, underutilization of effective services such as pulmonary rehabilitation, and inadequate self-management behaviors. ${ }^{89}$ Commonly reported consequences include compromised functional capacity, disease-related psychosocial disorders, and poor quality of life. ${ }^{10}$ These markers of suboptimal COPD care quality can be partly attributed to gaps in interprofessional collaborative practice and patient-provider coproduction. For example, delayed diagnosis and treatment initiation can result from oversights in referral practices and care coordination between primary care providers and pulmonary specialists. Inadequate selfmanagement behaviors are frequently associated with deficient patient education and counseling, as well as ineffective patient-provider communication. ${ }^{11}$

The hallmarks of coproduction among patients and providers include shared treatment goal-setting and decision-making, mutual understanding of the patient's disease-related knowledge and needs for education, and commitments to respectful and productive communication. ${ }^{11}{ }^{12}$ Evidence is mounting to support the view that patients who are engaged as partners in their health care experience better outcomes at lower costs. ${ }^{13}$ Moreover, patients' perceptions of the quality of their COPD care are correlated with the strength of coproductive relationships with their providers. ${ }^{11}{ }^{12}$ To be effective in promoting coproduction, however, QI initiatives must be informed by evidence and insights into the extent to which relationships between COPD patients and providers, as well as among members of interprofessional care teams, are indeed coproductive.

Comprehensive studies are lacking in the literature on coproduction in COPD. Findings from two recent studies indicated disconnects between patient and provider perceptions of patients' knowledge needs and preferred methods of education, as well as beliefs about the effects of smoking on disease etiology and progression. ${ }^{14}{ }^{15}$ Through the QI interprofessional continuing education (IPCE) program reported in this article, we sought to identify alignments and mismatches in patients' and providers' goals, perceptions, attitudes, and behaviors regarding various aspects of COPD care quality.

\section{BASELINE MEASUREMENT}

After obtaining independent institutional review board (IRB) approval, we identified regions of the U.S. with a high prevalence of people with COPD and a high density of primary care practices treating COPD patients. To identify these regions, we used the 2010 U.S. Census, surveillance data from the Centers for Disease Control and Prevention, and the American Medical Association Physician Masterfile. Crossover data from these sources were used to calculate a composite score for ranking the 48 contiguous states by population, COPD prevalence, and density of primary care practices. We identified the top 10 states by highest-ranking composite scores and, from these states, selected 1,257 primary care practices through ICD-9 codes for COPD. We used electronic and telephonic communications to recruit a final cohort of 30 primary care practices with interprofessional clinical staff. From each practice, a physician, nurse practitioner, or physician assistant was enrolled as the primary participant in the program, and each provider was encouraged to engage his/her entire treatment team in the project. The enrolled providers reported having office visits with an average of 18 patients with COPD per week.

Each of the 30 primary care providers invited three of their patients with COPD to participate in a "tethered" patient-provider survey study. (Throughout this article, the tethered group refers to patients who received primary care from the 30 providers in the QI cohort.) The surveys included items that prompted patients and providers to indicate their respective COPD treatment goals, perceptions about the disease symptoms and its effects on quality of life, assessment of patients' abilities to recognize and respond to COPD exacerbations, views on patient barriers to medication adherence, attitudes and practices regarding shared decision-making, and other aspects of COPD care quality. In addition to the 90 patients in the tethered cohort, a separate external group of 125 patients, who were recruited from COPD advocacy groups across the U.S., completed the patient surveys (Patient advocacy groups are organizations that provide services to patients with specific diseases or 
disorders; the services may include awareness-building activities, educational programs, and various forms of support for individuals affected and their caregivers).

The surveys were designed to identify alignments and mismatches in patients' and providers' responses to linked items. Our systematic approach to tethered survey development begins with a literature review of validated survey instruments and studies on patient-provider perceptions, and incorporates their findings to create surveys that are easily understood by patients and meaningful to providers. We develop survey items at the Flesch-Kincaid 5th - 6th grade level based on the Flesch Reading Ease score, which evaluates readability based on syllables, numbers of words per sentence, and other elements for patients. Survey instruments are included in our IRB protocol. Patients and providers complete surveys online or through a mobile application. Our rigorous review process includes multiple internal and external reviews, conducted by patients, interventional mapping consultants, expert faculty in the disease state, practicing clinicians, and research professionals. Not only are our surveys tailored to the population and setting, their implementation is managed by our QI specialists who are dedicated to guiding participants through the project from beginning to end.

As described in the following section, the patientprovider survey findings were individually presented to the 30 primary care practices as an educational intervention during a private feedback session conducted with each practice. In addition, providers in the 30 practices participated in a series of QI-focused online/mobile educational activities on COPD, which were also disseminated to a national audience of more than 1,000 clinicians including physicians, nurse practitioners, physician assistants, nurses, pharmacists, and case managers.

Key baseline findings from the patient surveys are summarized as follows:

- The majority of patients $(69 \%)$ indicated that they are not completely satisfied with their COPD treatment plan.

- Despite reporting that cigarette smoking is the most likely cause for their COPD, $87 \%$ of patients indicated that they currently smoke at least 1 cigarette per week.

- In response to a question about the sufficiency of information received from their COPD health care team $(1=$ none; $3=$ the right amount; $5=$ too much $)$, patients' mean rating for information and assistance on smoking cessation was 2.3.

- Although a large proportion of the patients reported feeling confident in discussing questions about COPD with their physicians $(80 \%)$, relatively few patients expressed confidence in such discussions with other members of their COPD care teams, including nurses $(32 \%)$ and other health care professionals $(24 \%)$.

- Patients gave low to moderate ratings (4.0-7.6 on a 10-point scale) to different members of interprofessional care teams for their understanding of the impact of COPD on emotional health and quality of life.

The surveys included an open-ended item asking patients for the main messages they would like to give providers about how to improve COPD care quality. The general themes of these messages are summarized as follows:

- Improve listening and communication skills

- Demonstrate greater understanding and compassion about the effects of COPD

- Provide education on lifestyle modification, new treatments, self-care, and other important aspects of COPD care

- Work cooperatively with the patient's other health care providers

- Involve patients in treatment and care decisions

- Direct patients to community education and support resources

Supplementary Table 1 presents examples of patients' open-ended comments categorized by these themes.

Baseline assessments for the 30 providers were qualitative, derived through reviewing transcript notes taken during the survey feedback sessions. Key themes and observations derived from discussions during the sessions are summarized as follows:

- Across providers, there was considerable variation in provision of patient education on essential self-care topics such as recognizing exacerbations and taking appropriate action to ensure timely treatment.

- Many providers reported that they educated patients about COPD causes, risks, symptoms, and treatment; however, few providers offered education and counseling on patient-centered interests and outcomes such as strategies for emotional coping and improving quality of life.

- Many providers acknowledged a lack of training and experience in counseling to address issues of emotional health and quality of life for patients with COPD.

- There was considerable variation in attitudes and practices regarding shared decision-making. Some providers commented that they take full responsibility for treatment decision-making because they "know what is best" for their patients. At the other end of the spectrum, providers expressed the view that they are patient advocates who regularly encourage shared decision-making. Reported barriers to effective shared decision-making included a lack of training and experience in cultural competence.

- Several of the primary care providers cited suboptimal communication with pulmonology specialists as a barrier to optimal COPD care quality.

Our qualitative analysis indicated that the different perspectives among providers were not systematically associated with their ages, gender, geographic location, or other demographic factors.

For the series of online/mobile educational activities on COPD, we developed a pre- and post-program survey 
to assess providers' attitudes and barriers to patientcentered COPD care and self-assessed ability to perform key skills in coproductive relationships with patients. These activities were to designed to improve providers' confidence and competence in COPD assessment as well as in patient counseling and shared decisionmaking. At the time this article was published, baseline surveys for the ongoing program had been completed by more than 1,000 interprofessional providers in addition to the 30 QI cohort providers. The pre- and postprogram surveys included items for self-assessing abilities to perform various patient-centered and coproductive skills. On the pre-program survey, relatively low proportions of providers in the QI cohort reported strong agreement with statements indicating high levels of confidence in assessing COPD prognosis and in counseling patients about topics of COPD treatment (30\%) and quality of life through referrals to case management, skilled nursing, and palliative care $(10 \%)$. (See Supplementary Table 2.) Respondents reported that their greatest barriers to providing optimal COPD care were patients' continued smoking (72\%), nonadherence to treatment plans (69\%), and lack of understanding about COPD progression (43\%).

\section{DESIGN}

The main educational components in this program were (1) 30 live and Internet-based performance improvement courses in which the 30 primary care practices received feedback on patient-provider survey findings; (2) an Internet-based (online/mobile) simulation course on patient-centered and interprofessional COPD care; (3) a set of 3 nonaccredited checklists for aligning COPD care with evidence-based guidelines and national quality measures; and (4) 2 educational tools specifically designed for patients.

During the private feedback courses, graphs were presented that compared de-identified and aggregated survey responses for (1) the participating physician, nurse practitioner, or physician assistant; (2) the provider's patients in the tethered cohort; (3) all patients in the tethered cohort, which enabled benchmark comparisons with peer providers; and (4) and patients in the COPD advocacy groups. In presenting the survey findings, the clinician-educator pointed out alignments and mismatches between the provider's responses and those of the different patient groups. The clinician-educator engaged the provider and participating clinical team members in reflection and discussion of targeted action plans for resolving identified mismatches. A moderator took notes on the discussions. Providers were also able to view the survey findings on a personalized online dashboard. At the conclusion of each course, providers were prompted to identify a personalized action plan for improvement in coproductive COPD care.
The rationale for the private feedback session was based on principles and evidence for audit and feedback as an intervention in continuing education programs for health care professionals. In a meta-analysis of 140 studies on the effects of audit and feedback education, significant improvements in clinical practice were observed. ${ }^{16}$ Our adaptation and implementation of this method were also informed by supporting rationale from a white paper developed by the Agency for Healthcare Research and Quality ${ }^{17}$ and our application of feedback in previous successful QIE programs. ${ }^{18-22}$ The essential premise of this method is that, through reviewing quantitative and qualitative data that directly reflect their clinical performance, health care professionals can reinforce strengths and develop targeted strategies for overcoming weaknesses. Data indicating alignments and mismatches in aspects of patientprovider coproduction enable opportunities to effectively negotiate treatment goals and align practices with patient-centered measures of care quality.

The Internet simulation course was designed as a follow-up intervention to reinforce lessons learned and provide evidence-based information to support action plans developed in the feedback sessions. It was also designed to engage and educate the interprofessional treatment team in unified learning. This course was accredited for 1.5 hours for physicians and physician assistants by the Accreditation Council for Continuing Medical Education (ACCME); for nurse practitioners by the American Association of Nurse Practitioners (AANP); for pharmacists by the Accreditation Council for Pharmacy Education (ACPE); for nurses by the American Nurses Credentialing Center (ANCC); and for case managers by the Commission for Case Manager Certification (CCMC).

The simulation demonstrated case-based approaches to effective interprofessional collaborative practice in COPD and coproductive strategies for overcoming barriers to optimal COPD care and treatment outcomes. Reinforcement of the interprofessional team was established through the IPCE design of the simulation and multi-accreditation. Additional reinforcement of these skills and strategies was afforded by the series of COPD care checklists and patient education tools. The checklists contained items for (1) guideline-directed COPD diagnosis and assessment; (2) educating patients about COPD, lifestyle modifications, medication use, exacerbation prevention strategies, and follow-up visits with providers; and (3) performing NQS-related measures for clinical care, patient safety, and community/population health. The patient tools comprised a self-assessment questionnaire and a self-management tool.

The simulation course and the COPD care checklists were packaged in an online/mobile toolkit that facilitated access to the 30 primary care providers, interprofessional members of their practices' clinical teams and, as of the publication date for this article, a national audience of more than 1,000 primary care and specialty providers. 
In analyzing the patient-provider survey data, we compared percentages of responses to linked items for the participating provider and the tethered and advocacy patient groups. This method enabled identification of alignments and mismatches in COPD-related goals, perceptions, attitudes, and behaviors. The design facilitated investigation of potential influences of patient participation in advocacy group activities that might improve coproductive skills. The analysis was guided by our tethered patient-provider model of learning, which is based on a framework of coproductive aims for patient and provider education, ${ }^{23}$ adopted from the HIMSS Foundation and National eHealth Collaborative's patient engagement model. ${ }^{24}$ Outcome domains were identified by the framework of Moore et al., such that pre- to postactivity improvements self-reported competence and performance were categorized at levels 4 and $5 .^{25}$

\section{STRATEGY}

The project's three sets of educational components-the private feedback courses, the case-based simulation activity, and the COPD care toolkit-instantiate our strategy for supporting the participating providers in improving competencies in essential interprofessional and coproductive aspects of COPD care. In addition, the series of interventions facilitated outcomes to inform our development of a phase 2 QI education project that we began in early 2016. As described in this article, the feedback course was designed to guide providers in developing a personalized action plan to close identified gaps in coproductive aspects of COPD care. In the successive educational activities, this action plan served as input to orient participants to targeted, individual learning objectives and as impetus toward program completion. During the online/mobile activities, providers completed course evaluations intended to measure improvement in knowledge, attitudes, beliefs, and competence related to evidence-based treatment and coproductive COPD care. Data used as input for the improvement cycles are presented in Supplementary Table 2.

\section{RESULTS}

\section{Demographics}

For the patients in the tethered $(\mathrm{n}=90)$ and advocacy groups $(\mathrm{n}=125)$, mean ages were 64 and 67 years, respectively; mean ages at COPD diagnosis were 54 and 56 years; and mean durations since diagnosis were 9 and 11 years. A substantial proportion of patients in the tethered group (39\%) and advocacy group (50\%) reported having had respiratory symptoms for more than two years before they were diagnosed with COPD. A large percentage of patients in the tethered and advocacy groups reported that they were in advanced stages of COPD, either Stage $3(30 \%$ and $36 \%$, respectively) or Stage 4 ( $16 \%$ or $42 \%$, respectively).
As derived from the patient-provider surveys, key mismatches and alignments in coproductive aspects of COPD care are summarized as follows.

\section{COPD Testing Frequency}

Patients and providers were asked to indicate how often spirometry or lung function testing was performed. Whereas $36 \%$ and $34 \%$ of patients in the tethered and advocacy groups, respectively, reported that they never or rarely receive these tests, $83 \%$ of the providers reported that they perform the tests regularly.

\section{Shared Treatment Goals}

A survey question asked patients and providers to indicate their most important goals for COPD treatment. Participants were instructed to select all goals that they deemed important from a list of five goals. The survey results indicated a common pattern for three of the treatment goals: managing symptoms, preventing exacerbations, and preventing hospitalizations. As shown in Supplementary Table 2, the majority of providers rated all three of these goals as highly important; however, relatively low percentages of patients in the tethered and advocacy groups gave high ratings.

\section{Agreement on Treatment Plan}

Among patients in the tethered and advocacy groups, respectively, $81 \%$ and $52 \%$ reported having agreed with their providers on a COPD treatment plan, whereas $57 \%$ of providers indicated this agreement. Across the tethered and patient advocacy groups, $86 \%$ and $61 \%$ of patients reported that they did not miss a prescribed dose during this period. However, only $59 \%$ of providers estimated that their patients were fully adherent.

\section{Perceived Barriers to Medication Adherence}

Compared with patients in the tethered and advocacy groups, a smaller proportion of providers attributed medication non-adherence to patients' having trouble remembering to take their medications (see Supplementary Table 2). Relatively fewer patients than providers indicated that a main barrier to adherence is patient beliefs that COPD treatment plans are not effective in achieving goals. A similar pattern was observed for attributing non-adherence to lack of patient insurance coverage.

\section{Perceived Impact of COPD on Quality of Life}

In response to a survey item addressing the impact of COPD on emotional aspects of quality of life, a large proportion of patients in the tethered and advocacy groups associated their disease with experiences of fatigue $(61 \%$ and $64 \%)$, frustration $(46 \%$ and $59 \%)$, and feelings of being overwhelmed (38\% and 50\%). Providers estimated, on average, that only $33 \%$ of their patients are emotionally affected by COPD. During the private feedback sessions, a number of providers acknowledged the importance of addressing patients' 
emotional needs, but they admitted a lack of training and competence in the assessment, communication, and management skills necessary to adequately support patients.

\section{Most Important Topics for COPD Education}

Patients and providers gave similar high ratings for the importance of various topics for COPD education, including topics of treatment options, side effects, importance of adherence, and symptom management. However, a greater proportion of providers $(79 \%)$ than patients in the tethered and advocacy groups $(45 \%$ and $17 \%$ ) rated smoking cessation assistance as a highly important topic. For most of the topics, with the exception of smoking cessation assistance, the advocacy patient group's ratings were higher than those of the tethered patient group and closer to those of the providers.

\section{Improvement in Knowledge, Attitudes, Beliefs Related to Coordinating COPD Care}

As indicated in self-assessment evaluations following the on-demand online and mobile educational activities, the 30 primary care providers in the cohort significantly improved their knowledge, attitudes, and beliefs related to coproductive COPD care. All cohort providers expressed confidence in being able to achieve their personal action plans over the next six months to improve the quality of their COPD patients' care. From pre- to post-program, there was a $55 \%$ increase in providers' self-reported ability to discuss referrals for case management, skilled nursing, and palliative care with COPD patients; a $32 \%$ increase in recognizing benefits of pulmonary rehabilitation; and a $36 \%$ increase in providers' self-rated ability to discuss COPD prognosis/progression and available pharmacologic options with their patients. Additional pre- and post-activity survey data indicating positive effects of the education are presented in Supplementary Table 2.

Improvements were also seen among the more than 1,000 interprofessional learners nationwide who accessed the online activities (43\% primary care; $12 \%$ physician specialists; $34 \%$ nurse case managers; $7 \%$ pharmacists; $4 \%$ physician assistants). There was a $43 \%$ increase in learners' self-reported ability to discuss referrals for case management, skilled nursing, and palliative care with COPD patients; a $25 \%$ increase in recognizing benefits of pulmonary rehabilitation; and a $47 \%$ increase in self-rated ability to discuss COPD prognosis/ progression and available pharmacologic options with COPD patients.

\section{LESSONS AND LIMITATIONS}

Primary objectives of this project were to assess gaps in coproductive COPD care and raise awareness and knowledge among patients, providers, and the interprofessional COPD team, as well as to inform the development of a comprehensive phase 2 QI education project on COPD. The following lessons learned and interpretations of the results address these objectives.

- The baseline data for primary care providers who participated in the online/mobile educational activities revealed needs for QI-focused continuing education on coproductive skills including communicating with patients about COPD progression and prognosis, treatment options, and referrals to specialists.

- The patient-provider survey data indicated key mismatches in perceptions of patients' receipt of important COPD assessment tests, patients' abilities to recognize exacerbations, patients' barriers to medication adherence, and the impact of COPD on quality of life. In addition, disconnects were identified in coproductive goal-setting and agreement on treatment plans. These disconnects reflect needs and opportunities for collaborative patient-provider education designed to promote accurate and timely recognition of symptoms, prompt implementation of action plans to avoid exacerbations, and shared understanding of treatment goals and barriers to achieving them.

- Alignments between patient-provider survey responses were observed for the importance of many, but not all, topics of patient education. In addition, assessment of overall quality of COPD care, there was agreement between patients' overall ratings of "good" or "very good" and providers' estimates of patients' ratings.

- For many survey items, responses were more closely aligned for patients in the advocacy group and providers compared with patients in the tethered group and providers. This observation supports inclusion of advocacy initiatives in QI education programs for COPD.

Given the project's focus on primary care providers, the outcomes are not generalizable to pulmonology specialists. However, a large proportion of patients with COPD in the U.S. are treated and managed in primary care settings. The project's outcomes are especially relevant in addressing interprofessional aspects of COPD care, including needs for appropriate and timely referrals to specialists and effective care coordination and transitions. As planned, the primary care providers who participated in the patient-provider survey study and feedback sessions practiced in areas of the U.S. with a high prevalence of COPD and high practice density. Thus, generalizability to primary care providers in less populated areas with fewer COPD patients, including some rural communities, is also limited.

This project was designed to identify interprofessional and coproductive correlates of COPD care quality in the context of a continuing education program, which provides standardized educational methods and measures under regulated accreditation criteria to engage clinicians in continuing professional development. Thus, the 
project was not intended to implement formal improvement methods such as PDSA. Moreover, it would have been ideal to collect more data points and extend the project over a longer period of time in order to assess its sustainability. However, as intended, the project has informed the design and development of a larger-scale phase 2 project in which chart data on providers' adherence to quality measures for COPD and patient-centered care will be collected in multiple improvement cycles over an extended period. The phase 2 program began in early 2016 and will end in 2017. Results are intended for future publication.

This pragmatic QI education project was not designed to determine the extent to which the three interventions were individually responsible for the improvements observed in the survey conducted for Internet-based (online/mobile) simulation course.

\section{CONCLUSION}

Through patient-provider surveys and assessments conducted in association with continuing education activities, we have identified key alignments and mismatches in patients' and primary care providers' goals, perceptions, attitudes, and behaviors regarding interprofessional and coproductive aspects of COPD care. The project outcomes are applicable to designing QI education interventions to support providers and patients in reinforcing coproductive alignments and in developing skills for communication, negotiation, and reconciliation to appropriately resolve mismatches that undermine high-quality care.

Acknowledgements The authors acknowledge James Mateka and Melany Vidret for coordinating the cohorts for this QI project; Laura Simone for contributing to the development of the patient-provider survey and educational content; and Jeffrey Carter and Matthew Weeks for contributing to the analysis of the project data.

Declaration of interests The authors represent PRIME Education, Inc., a health care education company that received an independent educational grant from AstraZeneca LP to conduct the quality improvement project described in this article. AstraZeneca LP had no role in the study design or execution, and the grant did not include support for writing this manuscript.

Ethical approval Independent institutional review board approval was granted before participants were recruited and enrolled in this program (Sterling IRB, Atlanta, GA, USA; IRB ID \#4991).

Open Access This is an open-access article distributed under the terms of the Creative Commons Attribution Non-commercial License, which permits use, distribution, and reproduction in any medium, provided the original work is properly cited, the use is non commercial and is otherwise in compliance with the license. See:

- http://creativecommons.org/licenses/by-nc/2.0/

- http://creativecommons.org/licenses/by-nc/2.0/legalcode

\section{REFERENCES}

1. Mannino DM, Braman S. The epidemiology and economics of chronic obstructive pulmonary disease. Proc Am Thorac Soc 2007;4:502-6.

2. Mannino DM, Homa DM, Akinbami LJ, Ford ES, Redd SC. Chronic obstructive pulmonary disease surveillance-United States, 19712000. Respir Care 2002:47:1184-99.

3. Ford ES, Murphy LB, Khavjou O, Giles WH, Holt JB, Croft JB. Total and state-specific medical and absenteeism costs of COPD among adults aged $=18$ years in the United States for 2010 and projections through 2020. Chest 2015;147:31-45.

4. Lindenauer PK, Pekow P, Gao S, Crawford AS, Gutierrez B, Benjamin EM. Quality of care for patients hospitalized for acute exacerbations of chronic obstructive pulmonary disease. Ann Intern Med 2006;144:894-903.

5. Mularski RA, Asch SM, Shrank WH, Kerr EA, Setodji CM, Adams $\mathrm{JL}$, Keesey J, McGlynn EA. The quality of obstructive lung disease care for adults in the United States as measured by adherence to recommended processes. Chest. 2006;130:1844-50.

6. Batalden M, Batalden P, Margolis P, et al. Coproduction of healthcare service. BMJ Qual Saf 2015 Sep 16. [Epub ahead of print]

7. Cramm JM, Nieboer AP. The changing nature of chronic care and coproduction of care between primary care professionals and patients with COPD and their informal caregivers. Int $J$ Chron Obstruct Pulmon Dis 2016;11:175-82.

8. Make B, Dutro M, Ryne P-R, et al. Undertreatment of COPD: a retrospective analysis of US managed care and Medicare patients. Int J Chron Obstruct Pulmon Dis 2012;7:1-9.

9. Ingebrigtsen TS, Marott JL, Vestbo J, et al. Characteristics of undertreatment in COPD in the general population. Chest 2013;144:1811-18.

10. Weldam SW, Lammers JW, Heijmans MJ, Schuurmans MJ. Perceived quality of life in chronic obstructive pulmonary disease patients: a cross-sectional study in primary care on the role of illness perceptions. BMC Fam Pract 2014;15:140.

11. Cramm JM, Nieboer AP. A longitudinal study to identify the influence of quality of chronic care delivery on productive interactions between patients and (teams of) healthcare professionals within disease management programs. BMJ Open 2014;4:e005914.

12. Cramm JM, Nieboer AP. Productive patient-professional interaction improves the well-being of chronically ill patients. Qual Life Res 2015;24:897-903.

13. Greene J, Hibbard JH, Sacks R, Overton V, Parrotta CD. When patient activation levels change, health outcomes and costs change, too. Health Aff (Millwood) 2015;34:431-7.

14. Hernandez P, Balter MS, Bourbeau J, et al. Canadian practice assessment in chronic obstructive pulmonary disease: respiratory specialist physician perception versus patient reality. Can Respir J 2013;20:97-105.

15. Menezes AM, Landis SH, Han MK, et al. Continuing to confront COPD International Surveys: comparison of patient and physician perceptions about COPD risk and management. Int J Chron Obstruct Pulmon Dis 2015;10:159-72.

16. Ivers N, Jamtvedt G, Flottorp S, et al. Audit and feedback: effects on professional practice and healthcare outcomes. Cochrane Database Syst Rev 2012;6:CD000259.

17. Agency for Healthcare Quality and Research. Private Performance Feedback Reporting for Physicians http://www.ahrq.gov/ professionals/clinicians-providers/resources/privfeedbackgdrpt/index. html.

18. Sapir T, Moreo K, Carter J, Greene L, Patel B, Higgins PDR. Continuing medical education improves gastroenterologists' compliance with inflammatory bowel disease quality measures. Dig Dis Sci 2016 Feb 12. [Epub ahead of print]

19. Sapir T, Rusie E, Greene L, Yazdany J, Robbins ML, Ruderman EM, Carter J, Patel B, Moreo K. Influence of continuing medical education on rheumatologists' performance on national quality measures for rheumatoid arthritis. Rheumatol Ther 2015; doi:10.1007/s40744-015-0018-7.

20. Moreo K, Sapir T, Greene L. Applying quality improvement into systems-based learning to improve diabetes outcomes in primary care. BMJ Qual Improv Report 2015;4.

21. Greene L, Moreo K. Quality improvement education to improve performance on ulcerative colitis quality measures and care processes aligned with National Quality Strategy priorities. BMJ Qual Improv Report 2015;4.

22. Greene L, Sapir T, Moreo K, Carter J, Higgins PD. Impact of quality improvement education on documented adherence to quality measures for adults with Crohn's disease. Inflamm Bowel Dis 2015;21:2165-71.

23. Moreo K, Goldenberg L. New patient-centered models of continuing education in the quest for quality. Available at: http://www.primece. com/downloads/new-patient-centered-models-2014.pdf.

24. Healthcare Information and Management Systems Society (HIMSS) Foundation and National eHealth Collaborative. Patient engagement framework. Available at: http://www.himss.org.

25. Moore DE, Green JS, Gallis HA. Achieving desired results and improved outcomes: integrating planning and assessment throughout learning activities. J Contin Educ Health Prof 2009;29:1-15. 
Erratum: Improving Interprofessional and Coproductive

Outcomes of Care for Patients with Chronic Obstructive Pulmonary Disease

Moreo K, Greene L, Sapir T. Improving Interprofessional and Coproductive Outcomes of Care for Patients with Chronic Obstructive Pulmonary Disease. BMJ Quality Improvement Reports 2016;5:u210329.w4679. doi:10.1136/bmjquality. u210329.w4679. The email address of the corresponding author Laurence Greene was updated since it was first published online.

Open Access This is an open-access article distributed under the terms of the Creative Commons Attribution Non-commercial License, which permits use, distribution, and reproduction in any medium, provided the original work is properly cited, the use is non commercial and is otherwise in compliance with the license. See:

- http://creativecommons.org/licenses/by-nc/2.0/

- http://creativecommons.org/licenses/by-nc/2.0/legalcode

BMJ Quality Improvement Reports 2016;5:210329.w4679. doi:10.1136/bmjquality.u210329.w4679corr1 